\title{
Bendamustine/Idelalisib/Rituximab Regimen
}

National Cancer Institute

\section{Source}

National Cancer Institute. Bendamustine/ldelalisib/Rituximab Regimen. NCI Thesaurus. Code C160549.

A chemoimmunotherapy regimen consisting of bendamustine, idelalisib and rituximab that can be used in the treatment of relapsed/refractory chronic lymphocytic leukemia (CLL). 